\title{
Successful Non-surgical Treatment of an Acute Calculous Cholecystitis in a Myeloma Patient with Covid-19: Case Report
}

\author{
Andy Sing Ong Tang ${ }^{1}$ (D) $\cdot$ Tze Shin Leong $^{1} \cdot$ Lee Ping Chew ${ }^{1} \cdot$ Hock Hin Chua $^{2}$ \\ Accepted: 25 January 2021 / Published online: 29 January 2021 \\ (C) The Author(s), under exclusive licence to Springer Nature Switzerland AG part of Springer Nature 2021
}

\begin{abstract}
COVID-19 has a wide spectrum of clinical phenotypes. While fever and cough are the most common symptoms, abdominal pain is rarely reported. We report the first case of COVID-19 pneumonia in an elderly patient with multiple myeloma (MM), complicated by acute calculous cholecystitis (ACC). A 73-year-old gentleman with underlying IgG kappa MM, presented with fever, cough and dyspnoea. His nasopharyngeal swab was positive for SARS-CoV-2. Piperacillin/tazobactam and oral hydroxychloroquine were started in addition to granulocyte colony-stimulating factor (GCSF) support due to neutropenia. Patient deteriorated on day 5, requiring high flow oxygen support. Dexamethasone, low molecular weight heparin prophylaxis and one dose of intravenous immunoglobulin were given. Despite negative swab on day 10, he developed ACC on day 17. With antibiotics and supportive care, patient showed full recovery without any surgical intervention. It remains elusive whether the gallbladder might be vulnerable to COVID-19, necessitating further validation via prospective studies.
\end{abstract}

Keywords COVID-19 $\cdot$ Myeloma $\cdot$ Cholecystitis

\section{Background}

Since its first emergence in late 2019, the novel coronavirus pneumonia, named coronavirus disease 2019 (COVID-19) by the World Health Organization (WHO), has been a global pandemic, leading to a high death toll [1]. As COVID-19 has a wide spectrum of clinical phenotypes, it is likely that many patients are undiagnosed. This spectrum ranges from

This article is part of the Topical Collection on Covid-19

Andy Sing Ong Tang

andytangsingong@gmail.com

Tze Shin Leong

tzeshin2001@yahoo.com

Lee Ping Chew

leepingc@gmail.com

Hock Hin Chua

hhchua2009@gmail.com

1 Ministry of Health Malaysia, Haematology Unit, Department of Medicine, Sarawak General Hospital, Jalan Hospital, 93586 Kuching, Sarawak, Malaysia

2 Ministry of Health Malaysia, Infectious Disease Unit, Department of Medicine, Sarawak General Hospital, Jalan Hospital,

93586 Kuching, Sarawak, Malaysia severe life-threatening respiratory failure requiring ventilator support to a completely asymptomatic disease course, with diversity in clinical manifestations in between. While fever and cough are the most common symptoms, abdominal pain is rarely reported [2]. We report the first case of COVID-19 pneumonia in an elderly patient with multiple myeloma (MM), complicated by acute calculous cholecystitis (ACC).

\section{Case Report}

A 73-year-old gentleman, an ex-smoker, was diagnosed with symptomatic MM (immunoglobulin GK) in January 2019. At that time, a bone marrow aspirate showed $15 \%$ clonal plasma cells and multiple osteolytic bone lesions with compression fractures in thoracolumbar regions on radiography. Initial laboratory investigations showed normocytic, normochromic anaemia (haemoglobin $10.1 \mathrm{~g} / \mathrm{dL}$ ) with Rouleaux formation on full blood picture, inverse albumin-to-globulin ratio (globulin $109 \mathrm{~g} / \mathrm{L}$ ), corrected serum calcium of $2.3 \mathrm{mmol} / \mathrm{L}$ and lactate dehydrogenase (LDH) of $452 \mathrm{U} / \mathrm{L}$. Serum protein electrophoresis (SPEP) reported IgG Kappa paraproteinemia of $51.2 \mathrm{~g} / \mathrm{L}$ in the gamma region with immune paresis. The patient received few cycles of induction chemotherapy consisting of thalidomide and dexamethasone; however, it 
was complicated by several bouts of pneumonia infection requiring hospitalization. Subsequently, thalidomide was replaced by melphalan from January 2020 .

Few months later, the patient complained of fever with chills and rigors, productive cough and dyspnoea for several days. Patient denied any gastrointestinal symptoms with no abdominal pain. Upon assessment, patient appeared tachypnoeic with oxygen saturation of $93 \%$ under room air. Lung auscultation noted bilateral coarse crepitation. Chest Xray showed bilateral lower zone patchy ground glass opacities. Due to resource-restricted setting in a non-COVID hospital, computed tomography (CT) of the thorax was not performed during admission. In this COVID-pandemic era, patient was admitted to isolation ward as per local protocol, and standard COVID-19 workup was performed. Serial laboratory investigation results are shown in Table 1. The first nasopharyngeal swab was negative, but due to high clinical suspicion, a second swab was repeated which turned out to be positive for SARS-CoV-2, detected using qualitative real-time reverse transcriptase-polymerase chain reaction (qRT-PCR) assay. No COVID-19 close contacts or travelling history were identified.

As patient's full blood count (Table 1) showed neutropenia and lymphopenia, intravenous (IV) piperacillin/tazobactam and oral hydroxychloroquine were started in addition to granulocyte colony-stimulating factor (GCSF) support. Patient deteriorated on day 5 of hospitalization (second week since symptoms onset) with respiratory distress and desaturation, requiring high flow oxygen support $(15 \mathrm{~L} / \mathrm{min})$. In view of systemic inflammatory response possibly triggered by cytokine, dexamethasone $(4 \mathrm{mg})$ twice daily and low molecular weight heparin (LMWH) prophylaxis were initiated. One dose of intravenous immunoglobulin (IVIG; $0.4 \mathrm{mg} / \mathrm{kg}$ ) was also given. Patient showed remarkable improvement with oxygen titrating down to nasal prong $2 \mathrm{~L} / \mathrm{min}$ and cell count recovering.

Patient's swab was negative for SARS-CoV-2 on day 10 of admission. He developed constant pain (pain score 8/10) in the right upper quadrant of the abdomen with positive Murphy's sign and sluggish bowel sound, not resolved with proton pump inhibitor and analgesic on day 17 of hospitalization. ACC was subsequently confirmed by $\mathrm{CT}$ of the abdomen which showed markedly distended gallbladder with calculi within, gallbladder wall thickening, peri-cholecystic fat density with no biliary dilatation. IV piperacillin/tazobactam was restarted. All culture and sensitivity $(C \& S)$ samples failed to detect any bacteria, mycobacterium or fungal species. Patient showed full recovery without the need of surgical intervention. No recurrence of cholecystitis was noted after 6 months of follow-up.

\section{Discussion}

To the extent of our knowledge, only one case of acute cholecystitis possibly related to COVID-19 was reported [3]. The literature analysing the relationship between gallbladder disease and COVID-19 is scarce. We report the first case of

Table 1 Patient's laboratory results during hospitalization and post-discharge

\begin{tabular}{|c|c|c|c|c|c|c|}
\hline $\begin{array}{l}\text { Laboratory } \\
\text { parameter }\end{array}$ & $\begin{array}{l}\text { Reference } \\
\text { range }\end{array}$ & $\begin{array}{l}\text { On admission (Day } \\
\text { 1) }\end{array}$ & $\begin{array}{l}\text { Day } 5 \\
\text { (desaturation) }\end{array}$ & $\begin{array}{l}\text { Day } 10 \text { (swab } \\
\text { negative) }\end{array}$ & $\begin{array}{l}\text { Day } 17 \\
\text { (ACC) }\end{array}$ & $\begin{array}{l}\text { 2-month post- } \\
\text { discharge }\end{array}$ \\
\hline $\mathrm{Hb}, \mathrm{g} / \mathrm{L}$ & $13.0-17.0$ & 8.7 & 7.9 & 9.1 & 9.6 & 11 \\
\hline $\mathrm{WBC}, \times 10^{3} / \mu \mathrm{L}$ & $4.0-10.0$ & 0.41 & 1.21 & 2.72 & 5.77 & 6.6 \\
\hline $\mathrm{ALC}, \times 10^{3} / \mu \mathrm{L}$ & $1.0-3.0$ & 0.07 & 0.06 & 0.24 & 0.58 & 2.8 \\
\hline $\mathrm{ANC}, \times 10^{3} / \mu \mathrm{L}$ & $2.0-7.0$ & 0.27 & 0.93 & 1.77 & 3.96 & 2.7 \\
\hline Platelet, $\times 10^{3} / \mu \mathrm{L}$ & $150-410$ & 34 & 38 & 54 & 146 & 152 \\
\hline Creat, $\mu \mathrm{mmol} / \mathrm{L}$ & $60-120$ & 85 & 66 & 89 & 71 & 60 \\
\hline AST, U/L & $1-37$ & 16 & 17 & 47 & 15 & 15 \\
\hline ALT, U/L & $1-41$ & 4 & 4 & 10 & 24 & $<4$ \\
\hline Albumin, $g / L$ & $35-52$ & 32 & 30 & 30 & 32 & 37 \\
\hline Globulin, $\mathrm{g} / \mathrm{L}$ & $17-35$ & 42 & 40 & 38 & 38 & 42 \\
\hline D-dimer, $\mu \mathrm{g} / \mathrm{mL}$ & $<0.2$ & 0.85 & 0.68 & 0.50 & 0.77 & $<0.2$ \\
\hline $\mathrm{CRP}, \mathrm{mg} / \mathrm{L}$ & $<47.6$ & 705.3 & 923.4 & 376.1 & 166.6 & N/A \\
\hline $\mathrm{CK}, \mathrm{U} / \mathrm{L}$ & 39-308 & 30 & 35 & 27 & 32 & N/A \\
\hline $\mathrm{LDH}, \mathrm{U} / \mathrm{L}$ & $125-220$ & 255 & 308 & 343 & 283 & 318 \\
\hline Calcium, mmol/L & $2.2-2.7$ & 2.16 & N/A & N/A & 2.21 & 2.18 \\
\hline COVID-19 PCR & Negative & Positive & Positive & Negative & Negative & N/A \\
\hline
\end{tabular}

$A C C$ acute calculous cholecystitis, $H b$ haemoglobin, $W B C$ white blood cell, $A L C$ absolute lymphocyte count, $A N C$ absolute neutrophil count, $C r e a t$ creatinine, $A S T$ aspartate aminotransferase, $A L T$ alanine aminotransferase $C R P$ C-reactive protein, $C K$ creatinine kinase, $L D H$ lactate dehydrogenase, $N / A$ not available, $P C R$ polymerase chain reaction 
COVID-19 pneumonia in an MM patient who suffers from ACC after 17 days of hospitalization, highlighting our speculation about this manifestation to be a possible complication of COVID-19. However, the precise mechanism remains elusive, pending further studies that could support this hypothesis. COVID-19 is no longer a virus that only involves respiratory system, but it should be considered as a multi-organ disease [4].

COVID-19 has become common among geriatric population, particularly in those who are immunocompromised like cancer patients [5]. At present, there is only sparse data on MM patients affected with COVID-19 especially in geriatric population [6]. Patients with MM are commonly associated with the acquisition of secondary immunodeficiency, which is attributed, in addition to the disease, to adverse effects of myeloma treatment and repeated infections $[7,8]$. Patients with MM are at high risk for mortality from COVID-19 infection, which further complicates the overall management. We postulate that our patient's adaptive immune system is suppressed as commonly found in MM patients, which then leads to low levels of immunoglobulin production, further increasing susceptibility to COVID-19 infection and acute cholecystitis. This might explain the fact that our patient responded well to IVIG treatment with remarkable clinical improvement. The beneficial effect of IVIG therapy in our patient is in accordance with several studies which reported a significant decrease in the occurrence of major and clinically documented infections using an IVIG dosage of 0.25 to $0.4 \mathrm{~g} / \mathrm{kg}$ body weight $[9,10]$.

Studies have shown that patients with COVID-19 infection are prone to develop thrombosis, possibly due to the systemic inflammatory response triggered by pro-inflammatory cytokines [11]. Several studies have reported almost 50\% rate of thrombotic events among the hospitalized patients with COVID-19 pneumonia $[12,13]$. The fact that no thrombosis was observed in our patient treated with prophylactic dose of enoxaparin during hospitalization justifies the rationale to consider prophylactic anticoagulation in this subset of patient population which is in accordance with a published practical review [14].

Hydroxychloroquine was administered in our patient as it was thought to be beneficial in reducing mortality among COVID-19 infected patients in the early pandemic era. Our patient was diagnosed and treated as COVID-19 infection in May 2020, when hydroxychloroquine was still a therapeutic option. Recent literature has shown that hydroxychloroquine produces little or no reduction in the mortality of hospitalized COVID-19 patients. Furthermore, it causes several adverse events [15].

D-dimer greater than $0.5 \mu \mathrm{g} / \mathrm{mL}$ has been shown to be associated with severe infection in patients with COVID-19 [16]. The imbalance of coagulation and fibrinolysis in the alveoli could result from inflammatory cytokines, leading to activation of the fibrinolysis system, which then increase the D-dimer level [17]. Our patient's D-dimer level (Table 1) decreased from $0.85 \mu \mathrm{g} / \mathrm{mL}$ on admission to less than $0.2 \mu \mathrm{g} / \mathrm{mL} 8$ weeks post-recovery, highlighting the importance of monitoring D-dimer levels throughout the disease course.

ACC occurs in 10 to $20 \%$ of untreated patients with gallstone [18]. The bile is relatively sterile in healthy individuals, but gallstone disease might result in bacterial colonization in 20 to $70 \%$ of patients [19, 20]. Empiric treatment with piperacillin/tazobactam or a cephalosporin \pm metronidazole is recommended for moderate and severe acute cholecystitis irrespective of whether there is growth by culture [21]. Despite the fact that surgical intervention remains the treatment of choice for ACC, it has high morbidity and mortality in elderly population [22]. We consider our patient to have a high risk of perioperative complications as he is elderly with immune dysregulation due to underlying myeloma disease and he has concomitant COVID-19 pneumonia and ACC. It is at our discretion that antimicrobial therapy might be the treatment of choice in this challenging scenario.

\section{Conclusion}

Our case highlighted the challenge posed in managing a myeloma patient with COVID-19 infection complicated by ACC. It remains elusive whether the gallbladder might be vulnerable to COVID-19, necessitating further validation via prospective studies.

Acknowledgements The authors would like to thank the Director General of Health Malaysia for the permission to publish this paper. We would like to thank all the medical team members involved in treating this patient for their hard works during the COVID-19 pandemic.

Author Contribution ASOT was responsible for the study design, data collection and manuscript writing. TSL, LPC and HHC participated in data collection and contributed to case illustration and discussion. LPC and $\mathrm{HHC}$ were involved in the manuscript editing and language proofreading. All authors read and approved the final manuscript.

SN COMPREHENSIVE CLINICAL MEDICINE

\section{Declarations}

Ethical Approval This article does contain studies with human participant and was registered via National Medical Research Register Malaysia with a Research ID of NMRR- 20-2194-56866.

Informed Consent Informed consent was obtained from the patient. 
Conflict of Interest The authors declare that they have no conflict of interest.

\section{References}

1. World Health Organization (WHO) Coronavirus disease (COVID19) situation reports - weekly epidemiological update and weekly operational update. Available from https://www.who.int/docs/ default-source/coronaviruse/situation-reports/20201012-weeklyepi-update-9.pdf. Accessed on $13^{\text {th }}$ October 2020

2. Guan WJ, Ni ZY, Hu Y, Liang WH, Ou CQ, He JX, et al. Clinical characteristics of coronavirus disease 2019 in China. N Engl J Med. 2020;382(18):1708-20.

3. Ying M, Lu B, Pan J, Lu G, Zhou S, Wang D, et al. COVID-19 with acute cholecystitis: a case report. BMC Infect Dis. 2020;20:437.

4. Spuntarelli V, Luciani M, Bentivegna E, Marini V, Falangone F, Conforti G, et al. COVID-19: is it just a lung disease? A case-based review. SN Compr Clin Med. 2020;28:1-6.

5. Dai M, Liu D, Liu M, Zhou F, Li G, Chen Z, et al. Patients with cancer appear more vulnerable to SARS-COV2: a multicentre study during the COVID-19 outbreak. Cancer Discov. 2020;10(6):78391.

6. Bentivegna E, Luciani M, Spuntarelli V, Speranza ML, Guerritore L, Sentimental A, et al. Extremely severe case of COVID-19 pneumonia recovered despite bad prognostic indicators: a didactic report. SN Compr Clin Med. 2020;2:1204-7.

7. Tamura H. Immunopathogenesis and immunotherapy of multiple myeloma. Int J Hematol. 2018;107:278-85.

8. Susek KH, Gran C, Ljunggren HG, Alici E, Nahi H. Outcome of COVID-19 in multiple myeloma patients in relation to treatment. Eur J Haematol. 2020;00:1-4.

9. Raanani P, Gafter-Gvili A, Paul M, Ben-Bassat I, Leibovici L, Shpilberg O. Immunoglobulin prophylaxis in crhonic lymphocytic leukemia and multiple myeloma: systematic review and meta-analysis. Leuk Lymphoma. 2009;50(5):764-72.

10. Khalafallah A, Maiwald M, Cox A, Burns D, Bates G, Hannan T, et al. Effect of immunoglobulin therapy on the rate of infections in multiple myeloma patients undergoing autologous stem cell transplantation or treated with immunomodulatory agents. Mediterr $\mathrm{J}$ Hematol Infect Dis. 2010;2(1):e2010005.
11. Joly BS, Siguret V, Veyradier A. Understanding pathophysiology of hemostasis disorders in critically ill patients with COVID-19. Intensive Care Med. 2020;46:1603-6.

12. Zhang L, Feng X, Zhang D, Jiang C, Mei H, Wang J, et al. Deep vein thrombosis in hospitalized patients with COVID-19 in Wuhan, China: prevalence, risk factors, and outcome. Circulation. 2020;142(2):114-28.

13. Klok FA, Kruip MJHA, Van der Meer NJM, Arbous MS, Gommers DAMPJ, Kant KM, et al. Incidence of thrombotic complications in critically ill ICU patients with COVID-19. Thromb Res. 2020;191:145-7.

14. Pennica A, Conforti G, Falangone F, Martocchia A, Tafaro L, Sentimental A, et al. Clinical Management of adult coronavirus infection disease 2019 (COVID-19) positive in the setting of low and medium intensity of care: a short practical review. SN Compr Clin Med. 2020;29:1-6.

15. Wesley HS, Matthew WS, Lindsay ML, Jonathan DC, Derek CA, Roy GB, et al. Effect of hydroxychloroquine on clinical status at 14 days in hospitalized patients with COVID-19: a randomized clinical trial. JAMA. 2020;324(21):2165-76.

16. Yu HH, Qin C, Chen M, Wang W, Tian DS. D-dimer level is associated with the severity of COVID-19. Thromb Res. 2020;195:219-25.

17. Tang N, Bai H, Chen X. Anticoagulant treatment is associated with decreased mortality in severe coronavirus disease 2019 patients with coagulopathy. J. Thromb. Haemost. 2020;18:1094-9.

18. Strasberg SM. Acute calcolous cholecystitis. N Engl J Med. 2008;358:2804-11.

19. Ikeda I, Yanaga K, Kusne S, Fung J, Higashi H, Starzl TE. Sterility of bile in multiple-organ donors. Transplantation. 1990;49(3):653.

20. Yun SP, Seo H. Clinical aspects of bile culture in patients undergoing laparoscopic cholecystectomy. Medicine. 2018;97(26):e11234.

21. Gomi H, Solomkin JS, Schlossberg D, Okamoto K, Takada T, Strasberg SM, et al. Tokyo guidelines 2018: antimicrobial therapy for acute cholangitis and cholecystitis. J Hepatobiliary Pancreat Sci. 2018;25(1):3-16.

22. McGillicuddy EA, Schuster KM, Barre K, Suarez L, Hall MR, Kaml GJ, et al. Non-operative management of acute cholecystitis in the elderly. Br J Surg. 2012;99(9):1254-61.

Publisher's Note Springer Nature remains neutral with regard to jurisdictional claims in published maps and institutional affiliations. 